\title{
A map of the cosmic background radiation at 3 millimeters
}

ARTICLE in THE ASTROPHYSICAL JOURNAL - DECEMBER 1985

Impact Factor: 6.28 · DOI: 10.1086/184555 · Source: NTRS

CITATIONS

97

4 AUTHORS, INCLUDING:

Philip Lubin

University of California, Santa Barbara

333 PUBLICATIONS 8,191 CITATIONS

SEE PROFILE

George Smoot

Université Sorbonne Paris Cité

575 PUBLICATIONS 19,371 CITATIONS

SEE PROFILE
Thyrso Villela

National Institute for Space Research, Brazil 115 PUBLICATIONS 655 CITATIONS

SEE PROFILE 
The Astrophysical Journal, 298:L1-L5, 1985 November 1

(C) 1985. The American Astronomical Society. All rights reserved. Printed in U.S.A.

\title{
A MAP OF THE COSMIC BACKGROUND RADIATION AT 3 MILLIMETERS
}

\author{
Philip Lubin, Thyrso Villela, ${ }^{1}$ Gerald Epstein, and George Smoot \\ Space Sciences Laboratory and Lawrence Berkeley Laboratory, University of California, Berkeley \\ Received 1985 June 17; accepted 1985 August 13
}

\begin{abstract}
We present data from a series of balloon flights covering both the northern and southern hemispheres measuring the large angular scale anisotropy in the cosmic background radiation at $3.3 \mathrm{~mm}$ wavelength. The data cover $85 \%$ of the sky to a limiting sensitivity of $0.7 \mathrm{mK}$ per $7^{\circ}$ field of view. The data show a $50 \mathrm{\sigma}$ (statistical error only) dipole anisotropy with an amplitude of $3.44 \pm 0.17 \mathrm{mK}$ and a direction of $\alpha=11^{\mathrm{h}} .2 \pm 0.1$ and $\delta=-6.0 \pm 1.5$. A $90 \%$ confidence level upper limit of $7 \times 10^{-5}$ is obtained for the rms quadrupole amplitude. Flights separated by 6 months show the motion of Earth around the Sun. Galactic contamination is very small with less than $0.1 \mathrm{mK}$ contribution to the dipole and quadrupole terms. A map of the sky has been generated from the data.
\end{abstract}

Subject heading: cosmic background radiation

The isotropy of the cosmic background radiation is one of the fundamental tests of modern cosmological theories. The angular distribution has been measured on scales from arc seconds to $180^{\circ}$. The only variations seen are the dipole at large angular scales, presumably due to our motion, and perhaps an effect due to the scattering of the radiation by free electrons in galactic clusters at small angular scales (SunyaevZel'dovich effect). Neither of these effects is due to an intrinsic cosmological perturbation. The lack of any observed intrinsic anisotropy on any angular scale is disturbing and has contributed to recent modifications of the standard model such as the inflationary universe models, which are more consistent with observations (Bond and Efstathiou 1984; Vittorio and Silk 1984).

We have made a series of observations on the large-scale $\left(>7^{\circ}\right)$ anisotropy in the background radiation at $3.3 \mathrm{~mm}(90$ $\mathrm{GHz}$ ) wavelength. The instrument consists of a liquidhelium-cooled Schottky diode receiver which measures the temperature difference between two $7^{\circ}$ fields of view $90^{\circ}$ apart in the sky. The apparatus is flown from a balloon at an altitude of $30 \mathrm{~km}$. The instrument is described in detail in Lubin (1982), Lubin, Epstein, and Smoot (1983), and Epstein (1983).

The system has a sensitivity of about $13 \mathrm{mK} \mathrm{Hz}$ noise temperature of $130 \mathrm{~K}$, and a bandwidth of $600 \mathrm{MHz}$ with a beam width of $7^{\circ} \mathrm{FWHM}$. The gondola rotates with a period of about $100 \mathrm{~s}$. The rotation of the gondola and Earth yields a sky coverage of about $30 \%$ in one $12 \mathrm{hr}$ flight. An inherent instrument asymmetry is caused by the beam chopper, which consists of a sector of highly polished aluminum. The emissivity of a piece of metal with our polarization orientation is $\varepsilon=\left(4 \pi \delta / \lambda_{0}\right) \cos \theta$, where $\theta$ is the angle of incidence, $45^{\circ}$ for us, and $\delta$ is the skin depth. The conductivity of aluminum at the ambient flight air temperature of $240 \mathrm{~K}$ gives an

\footnotetext{
${ }^{1}$ Also Instituto de Pesquisas Espaciais/CNPq, Departamento de Astrofísica, São José dos Campos, SP, Brazil.
}

emissivity of $6 \times 10^{-4}$. The observed inflight asymmetry (offset) was $200 \mathrm{mK}$ giving an emissivity of about $8 \times 10^{-4}$, in relatively good agreement. The offset was stable to about 1 $\mathrm{mK} \mathrm{hr} \mathrm{h}^{-1}$ during the flight.

Calibration was performed inflight every 27 minutes. In the first two data flights, a small blackbody target was used. Though it provided good relative calibration, it was difficult to use as an absolute calibrator to better than about $5 \%$. On the third data flight (Brazil), it was replaced by a full beam calibrator which appeared to be capable of a $1 \%-2 \%$ calibration. Unfortunately, the gondola was lost in this last flight. Though the data were recovered, the full calibration accuracy has not been achieved due to the loss of the flight tape from the gondola tape recorder. The Moon has also been used as an inflight calibration source. In particular, during the last flight, the beam passed directly over the Moon, permitting a good comparison between the full beam calibrator and the Moon. The lunar emission has been analyzed using a model of the Moon due to Keihm (1984). The calibration using the Moon agrees with the calibration obtained using the target calibrator within $3 \%$.

The preflight and postflight calibrations were the same within $2 \%$ for all flights except the last flight where no postflight calibration was possible. The inflight calibration stability was $\pm 1 \%$ over all flights. The overall calibration uncertainty was taken as $5 \%$. Because of the high sensitivity of the experiment, this uncertainty dominates the error in the dipole amplitude, though it has negligible effect on the higher order moments (quadrupole, etc).

Galactic emission is a problem for large-scale anisotropy measurements because of the extended size of the Galaxy and uncertainty in modeling its emission. At long wavelengths $(\lambda>1 \mathrm{~cm})$ synchrotron and bremsstrahlung radiation dominate, while at short wavelengths $(\lambda<1 \mathrm{~mm})$ dust emission dominates. Typical Galactic emission as a function of wavelength is shown in Figure 1. This estimate is based on low-frequency ground-based surveys for the synchrotron and 


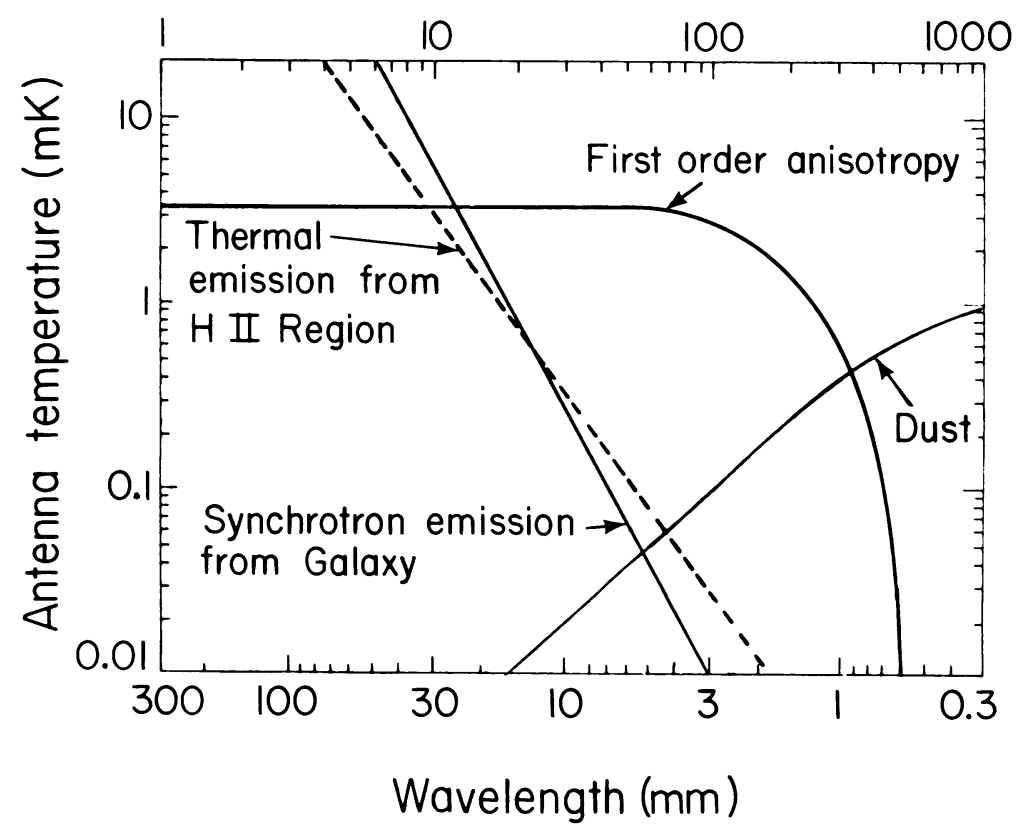

FIG. 1.-Estimated galactic emission. See Lubin and Villela (1984) for more details and comparison of recent measurements.

bremsstrahlung components and from submillimeter balloon measurements (Owens, Muehlner, and Weiss 1979) combined with our $3.3 \mathrm{~mm}$ data for the dust. A natural minimum, or window, occurs around $3 \mathrm{~mm}$ where our measurement was made. More recent measurements (Halpern 1983) suggest a somewhat flatter dust spectrum but with a minimum galactic contribution still near $3 \mathrm{~mm}$ (Lubin and Villela 1984).

At $30 \mathrm{~km}$, the residual atmospheric pressure is $10 \mathrm{mb}$. For our frequency and bandwidth, the vertical atmospheric emission is calculated to be $6 \mathrm{mK}$ (Lubin and Villela 1984) based on the line strengths of oxygen, water, and ozone and a model of their atmospheric distribution and temperature profile. To first order, the atmospheric contribution cancels out because the beam is symmetrical with respect to the zenith. Gondola wobble is measured with the magnetometers to be less than \pm 0 \% 5 , which combined with our offset gives a systematic error of less than $0.1 \mathrm{mK}$. The low sidelobe antenna and dual ground shields reduced the contribution of radiation from Earth to less than $0.1 \mathrm{mK}$.

The instrument has been flown four times. The first flight in 1981 July was on the gondola of D. Wilkinson of Princeton. Due to interference, this flight did not return useful data. The second flight, in 1981 November, was on the gondola of R. Weiss of MIT. The third flight, in 1982 April, and the fourth flight, in 1982 November, used our own gondola. The first three flights were from Palestine, Texas, at the National Scientific Balloon Facility. The fourth flight was from Cachoeira Paulista, SP, Brazil, at the Instituto de Pesquisas Espaciais. The sky coverage from the three data flights is shown in Figure 2 on a Hammer-Aitoff projection. Sky coverage is now $85 \%$ with coverage from $-68^{\circ}$ to $+77^{\circ}$ declination. The data from the Brazilian flight were taken from the telemetry tapes. The tapes were digitized and the data were calibrated using both the inflight observation of the Moon and the inflight target calibrator. The calibration was constant to $\pm 1 \%$ during the flight.

The dipole obtained from the Brazilian flight only is $\alpha=$ $11^{\mathrm{h}} .14 \pm 0.20, \delta=-8.2 \pm 3.0$ and $\Delta T_{A}=2.86 \pm 0.14 \mathrm{mK}$, in good agreement with the northern hemisphere data (Epstein 1983) of $\alpha=11^{\mathrm{h}} .25 \pm 0.10, \delta=-5.7 \pm 1.8$ and $\Delta T_{A}=$ $2.83 \pm 0.14 \mathrm{mK}$, where $\Delta T_{A}$ is the "antenna temperature." The fit to the dipole and quadrupole spherical harmonics is summarized in Table 1 . As can be seen, no significant quadrupole is present. The quadrupole fits imply a $90 \%$ confidence level upper limit of $7 \times 10^{-5}$ for the rms quadrupole component. The latest Princeton $25 \mathrm{GHz}$ maser data (Fixsen, Cheng, and Wilkinson 1983) and these and our previous data (Lubin, Epstein, and Smoot 1983) all are consistent with quadrupole upper limits at the $7 \times 10^{-5}$ level and appear to rule out the previous quadrupole reports. A fit to a galactic dust model with a $\operatorname{cosec} b$ ( $b$ is galactic latitude) distribution flattened at $b=5^{\circ}$ yields $44 \pm 11 \mu \mathrm{K}$ at the poles. While this appears to be significant, it should be interpreted cautiously. Excluding data within $5^{\circ}$ of the Galactic plane fit yields an insignificant $14 \pm 19 \mu \mathrm{K}$, indicating that the previous fit is dominated by emission near the Galactic equator as would be expected. Galactic emission appears to be a very small effect in these data as can be seen by the very small change in the multipole amplitudes when the Galactic plane is omitted.

The inferred velocity of the Sun is $382 \pm 25 \mathrm{~km} \mathrm{~s}^{-1}$ toward $\alpha=11^{\mathrm{h}} .2 \pm 0.1$ and $\delta=-6^{\circ} .0 \pm 1.5$ for a background radiation temperature of $2.7 \mathrm{~K}$. Using a Galactic solar velocity of $220 \mathrm{~km} \mathrm{~s}^{-1}$ toward $l^{\mathrm{II}}=90^{\circ}, b^{\mathrm{II}}=0^{\circ}$ gives a Galactic (Local Group) velocity of $V_{\mathrm{LG}}=550 \pm 40 \mathrm{~km} \mathrm{~s}^{-1}$ toward $\alpha=10^{\mathrm{h}} .7$ 


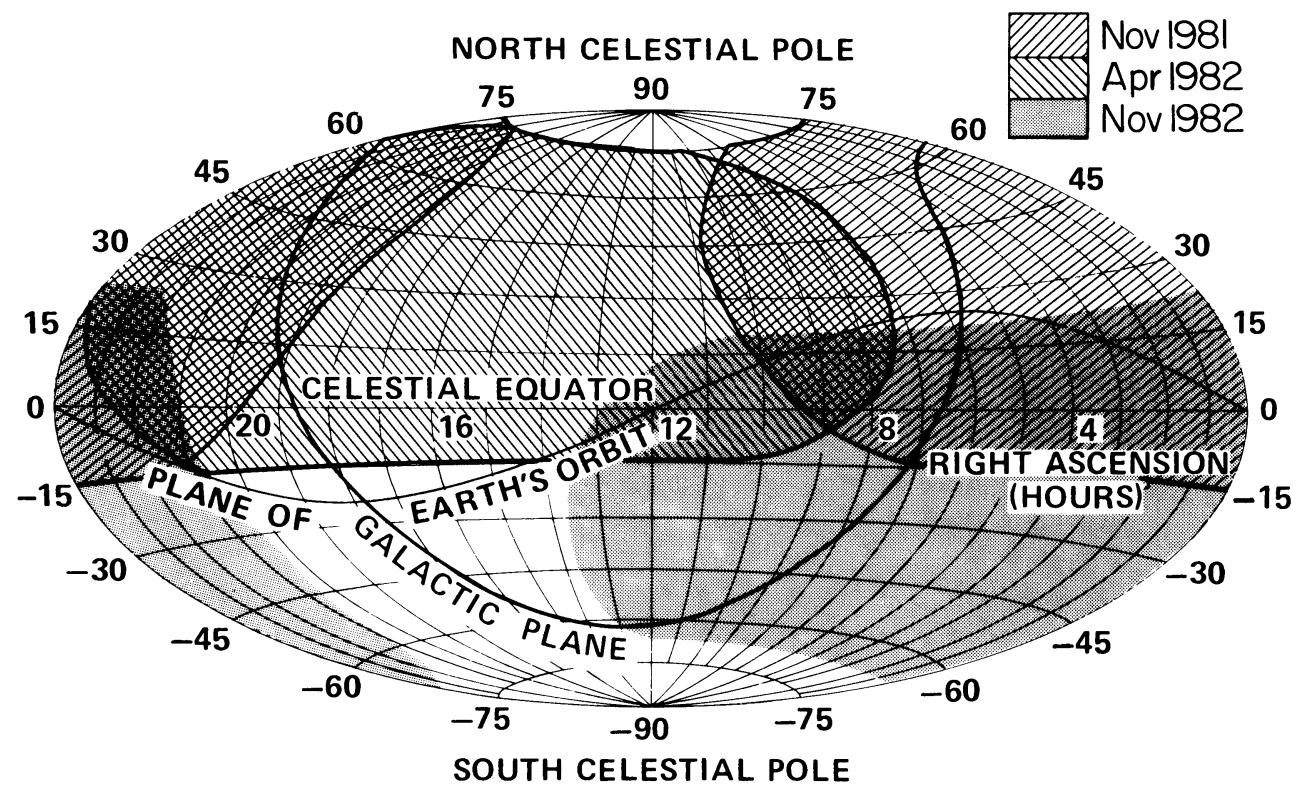

FIG. 2.-Sky coverage of three data flights by date

TABLE 1

SPherical HaRmonics Fits

\begin{tabular}{|c|c|c|c|c|c|}
\hline Fit & Coefficients $^{\mathrm{a}}$ & $\begin{array}{c}\text { All } \\
\text { Data }\end{array}$ & $\begin{array}{c}\text { Galactic } \\
\text { Cut } 5^{\circ}\end{array}$ & $\begin{array}{c}\text { Statistical } \\
\text { Error }\end{array}$ & $\begin{array}{l}\text { Total } \\
\text { Error }\end{array}$ \\
\hline \multirow[t]{3}{*}{ Dipole....$\ldots \ldots$} & $T_{x}$ & -3.36 & -3.36 & 0.07 & 0.17 \\
\hline & $T_{y}^{x}$ & 0.62 & 0.71 & 0.07 & 0.1 \\
\hline & $T_{z}$ & -0.37 & -0.36 & 0.06 & 0.0 \\
\hline \multicolumn{6}{|l|}{ Dipole and } \\
\hline \multirow[t]{8}{*}{ Quadrupole ... } & $T_{x}$ & -3.37 & -3.37 & 0.09 & 0.17 \\
\hline & $T_{y}$ & 0.63 & 0.69 & 0.09 & 0.0 \\
\hline & $T_{z}$ & -0.49 & -0.42 & 0.09 & 0.0 \\
\hline & $Q_{1}$ & 0.21 & 0.10 & 0.09 & 0.0 \\
\hline & $Q_{2}$ & 0.27 & 0.15 & 0.09 & 0.1 \\
\hline & $Q_{3}$ & 0.16 & 0.12 & 0.10 & 0.1 \\
\hline & $Q_{4}$ & -0.10 & -0.09 & 0.07 & 0.0 \\
\hline & $Q_{5}$ & 0.05 & 0.09 & 0.06 & 0.08 \\
\hline
\end{tabular}

NotE. - Values are $\mathrm{mK}$ thermodynamic temperature for $T=2.7 \mathrm{~K}$.

${ }^{\mathrm{a}} T=T_{x} \cos \delta \cos \alpha+T_{y} \cos \delta \sin \alpha+T_{z} \sin \delta+Q_{1}\left(3 \sin ^{2} \delta-1\right) / 2+$ $Q_{2} \sin 2 \delta \cos \alpha+Q_{3} \sin 2 \delta \sin \alpha+Q_{4} \cos ^{2} \delta \cos 2 \alpha+Q_{5} \cos ^{2} \delta \sin 2 \alpha$.

$\pm 0.3, \delta=-22^{\circ} \pm 5^{\circ}$. This gives an angle of $44^{\circ}$ between the center of the Virgo Cluster and $V_{\text {LG }}$

The orbital velocity of Earth around the Sun is roughly 30 $\mathrm{km} \mathrm{s}^{-1}\left(\beta \approx 10^{-4}\right)$ or $10 \%$ of Earth's velocity relative to the cosmic background radiation $\left(\beta \approx 10^{-3}\right)$. Flights separated by 6 months should be able to detect Earth's orbital motion as a 10\% change in the dipole. Our 1982 April and November 1982 flights clearly show this effect. The dipole difference vector between the flights is:

$\left(\Delta T_{x}, \Delta T_{y}, \Delta T_{z}\right)=(-0.30 \pm 0.14,0.40 \pm 0.14,0.08 \pm 0.14)$,

where the numbers are antenna temperature in $\mathrm{mK}$. The predicted difference based on Earth's orbital motion and assuming a $2.7 \mathrm{~K}$ background radiation temperature is $(-0.32,0.28,0.12)$ which is in good agreement.

The data set consists of approximately $10^{5}$ measurements of temperature differences distributed over $85 \%$ of the celestial sphere. To convert these different measurements into a map of the sky, we use a technique suggested by Gulkis and Janssen (1981). In essence, the idea is to find a sky distribution which minimizes

$$
\chi^{2}=\sum \frac{1}{\sigma_{i j}^{2}}\left(T_{i}-T_{j}-\Delta T_{i j}\right)^{2},
$$

where $T_{i}$ and $T_{j}$ are the map temperatures desired at pixels $i$ and $j$ and $T_{i j}$ is the measured temperature difference between pixels $i$ and $j$. In this procedure, the $T_{i}$ are free variables. Minimizing $\chi^{2}$ with respect to $T_{i}$ yields a set of $N$ linear equations, where $N$ is the number of pixel elements. These equations take the form:

$$
\sum_{1}^{N} \frac{1}{\sigma_{i j}^{2}}\left(T_{i}-T_{j}-\Delta T_{i j}\right)=0 .
$$

Our integrated beam pattern yields a solid angle of about $70 \mathrm{deg}^{2}$. This would give about 600 pixels for the full sky. We split each pixel into four parts giving about 2400 pixels for the full sky or 2000 pixels for our $85 \%$ sky coverage. Solving these 2000 linear equations is tantamount to inverting a 2000 by 2000 matrix. Instead of a direct matrix inversion, we use an iterative Gauss-Sidel inversion method which is efficient at iteratively inverting a large matrix. In addition, it converges rapidly, typically within 100 iterations. A subsidiary condition is also needed since we do not measure the absolute temperature. We chose to set the weighted average to 0 . Because the data are essentially taken at constant latitude, and each pixel is connected by observations to a few other pixels (the matrix 
is sparse and nearly singular), certain mathematical instabilities can occur in the map. For this reason, we have run numerous Monte Carlo simulations to test the stability of the maps. These tests show that on large scales the maps are a faithful representation of the data. A map made from the data is shown in Figure 3 (Plate L1) as a $\sin \delta$ projection which is an equal area projection. The map clearly shows the dipole anisotropy. The residual map with the dipole removed is shown in Figure 4 (Plate L2). No significant structure appears to be left. No corrections for Galactic emission have been made in the maps.

A summary of recent dipole measurements is shown in Figure 5. Recent measurements have been limited by about a $5 \%$ calibration error which is included in Figure 5. Combining the dipole measurements of the Princeton group at $25 \mathrm{GHz}$ and these data at $90 \mathrm{GHz}$ gives a spectrum which is consistent with a $2.7 \mathrm{~K}$ blackbody (Lubin and Villela 1984). Further analysis of our recently recovered Brazilian package may improve the calibration from $5 \%$ to $2 \%$ which would give a better idea of the spectrum (Villela 1985).

As large-scale anisotropy measurements are being fundamentally limited by systematic errors and particularly by Galactic emission, it is of interest to consider how much better the next generation of experiments will be. New technologies, such as superconducting receivers and ${ }^{3} \mathrm{He}$-cooled bolometers, may give an order of magnitude increase in system sensitivity. To take advantage of this, though, a much better understanding of Galactic emission, particularly at the millimeter wavelengths, will be necessary. With the recent launch of the Prognoz 9 satellite, space-based measurements are now possible. These have the advantage of no atmosphere and a longer observing time but generally do not use state-of-art techniques. Later in this decade the COBE satellite will be launched and should give us a much better understanding of Galactic emission and about a factor of 5 increase in sensitivity over the data presented here.

This work was supported by the California Space Institute under CS 48-81, by the National Aeronautics and Space Administration under NAGW-66, by the National Science Foundation under SPI 8166057, and by the US Department of Energy under DE-AC03-76SF00098. G. E. acknowledges the generous support of the Fannie and John Hertz Foundation. T. V. acknowledges the support and assistance of Conselho Nacional de Desenvolvimento Científico e Tecnológico $(\mathrm{CNPq})$, Brazilian Government research agency, Brazil, under 202372/83-FA, and Fundação de Amparo à Pesquisa do Estado de São Paulo (FAPESP), State of São Paulo research agency, Brazil, under 83/9834-1-Astronomia, during his research stay. The northern hemisphere balloon launches were conducted by NASA at the National Scientific Balloon Facility, Palestine, Texas. The southern hemisphere flight was supported and conducted by INPE/CNPq at its balloon launching facility at Cachoeira Paulista, SP, Brazil. This project would not have been fruitful without the fabrication assistance of Hal Dougherty and John Gibson and the encouragement and assistance of D. Wilkinson on whose gondola we flew for the first time. We appreciate the perseverance of the MIT group and especially of $\mathrm{R}$. Weiss in allowing us to fly our "MIT/Bezerkeley Lunar Lander." Special thanks go to P. Price, N. Boggess, R. Kubara, and also to the staff of the National Scientific Balloon Facility for their assistance and hospitality. Agradecemos os esforços e assistência do Departamento de Astrofísica do INPE durante o vôo de novembro de 1982 e o desenvolvimento subsequente deste trabalho.

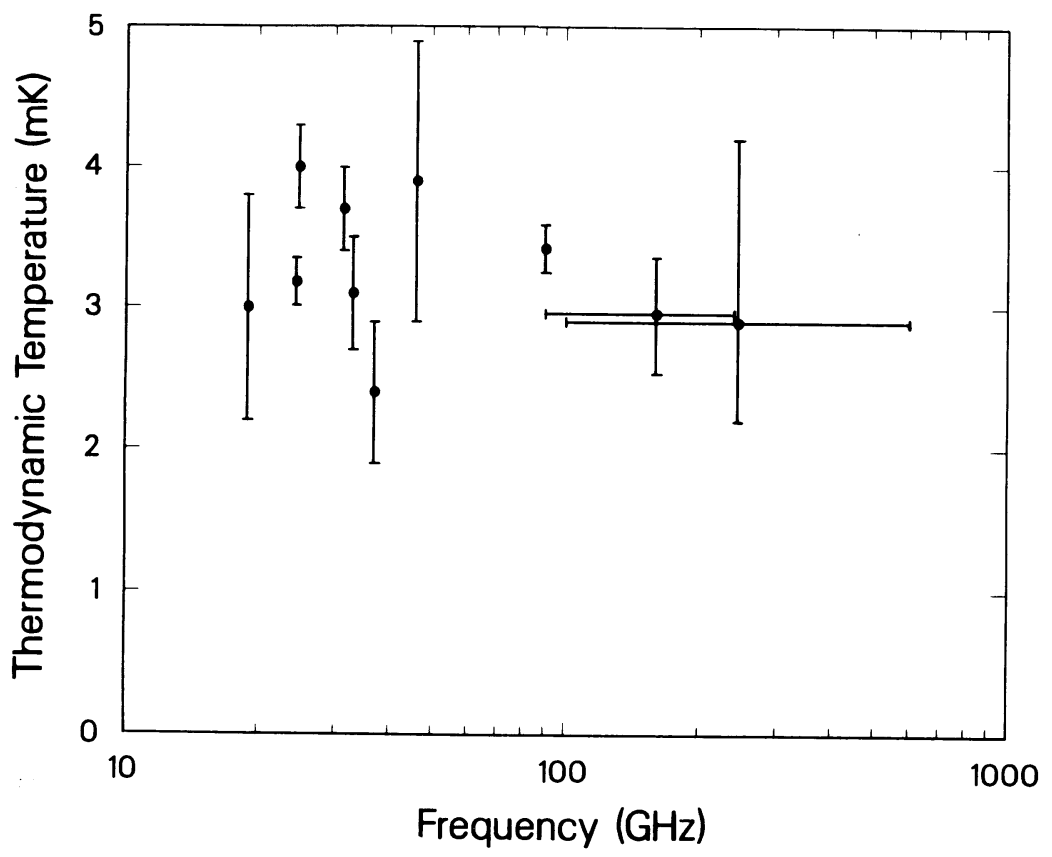

FIG. 5.-Recent dipole measurements vs. frequency. This work at $90 \mathrm{GHz}$. 


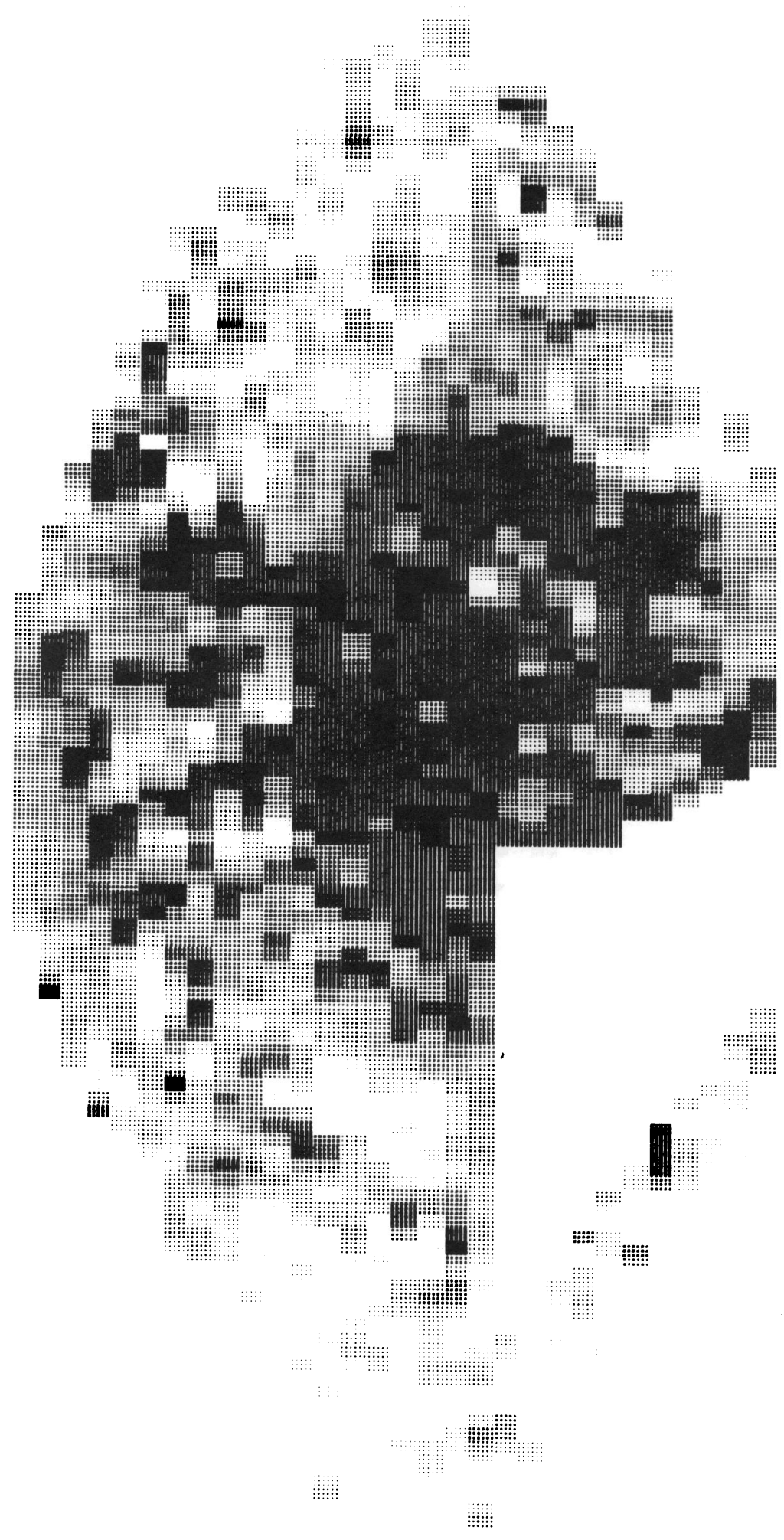

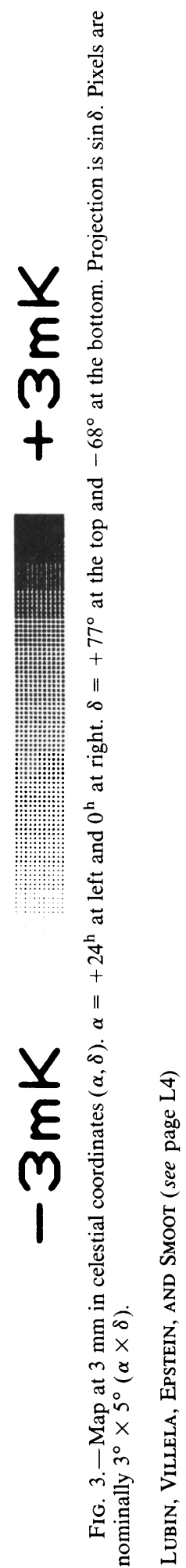



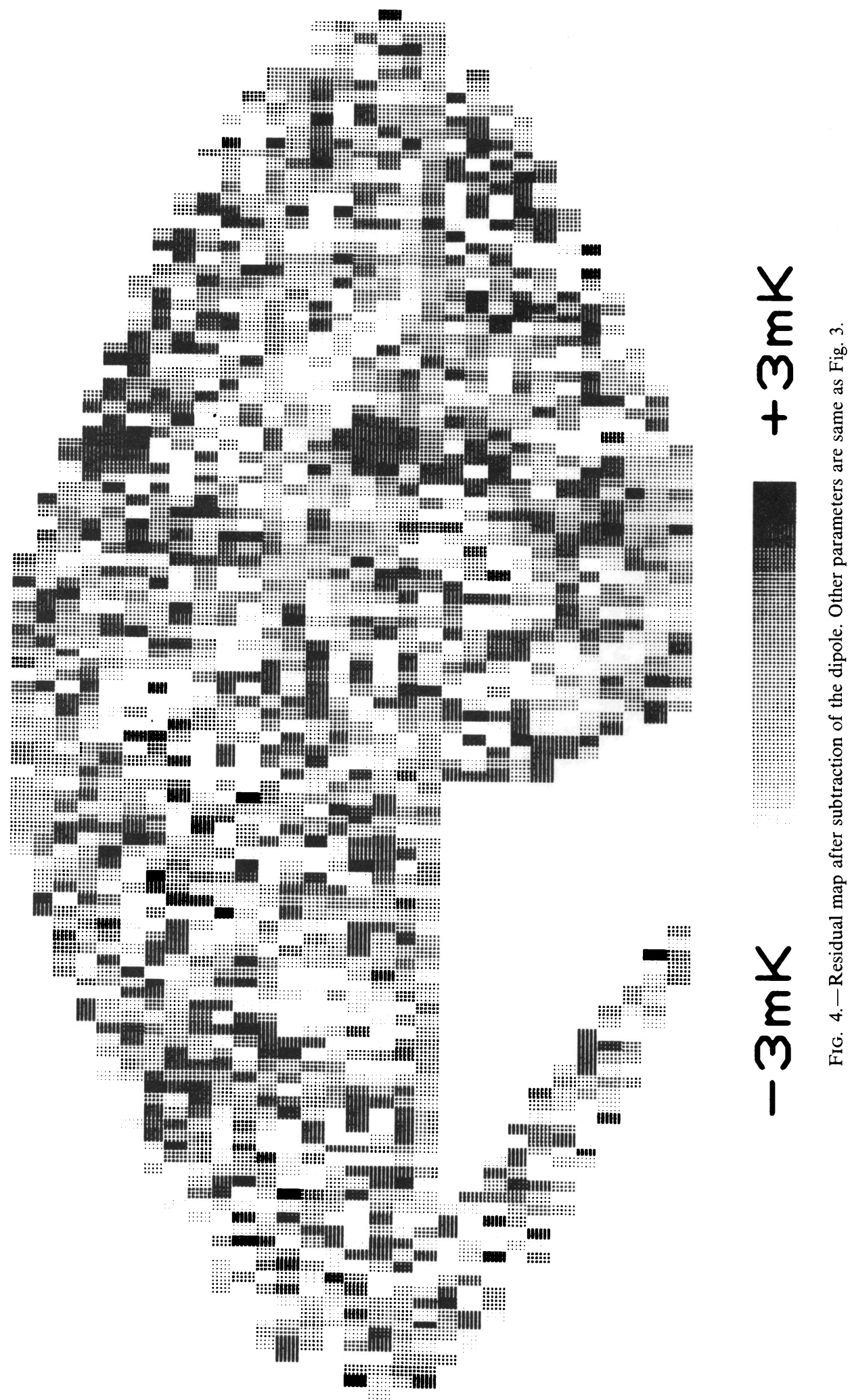

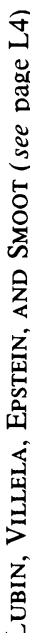




\section{REFERENCES}

Bond, J. R., and Efstathiou, G. 1984, Ap. J. (Letters), 285, L44.

Epstein, G. L. 1983, Ph.D. thesis, University of California, Berkeley.

Fixsen, D. J., Cheng, E. S., and Wilkinson, D. T., 1983, Phys. Rev. Letters, 50, 620.

Gulkis, S., and Janssen, M. 1981, NASA COBE Report 4004

Halpern, M. 1983, Ph.D. thesis, Massachussetts Institute of Technology.

Keihm, S. 1984, private communication.

Lubin, P. 1982, in Proc. of the International School of Physics "Enrico

Fermi," Course on Gamow Cosmology, Varenna, Italy, in press.
Lubin, P., Epstein, G., and Smoot, G. 1983, Phys. Rev. Letters, 50, 616. Lubin, P., and Villela, T. 1984, in Proc. of the Third Rome Meeting on Astrophysics, Rome, Italy, in press.

Owens, D. K., Muehlner, D. J., and Weiss, R. 1979, Ap. J., 231, 702.

Villela, T. 1985, Ph.D. thesis, in preparation.

Vittorio, N., and Silk, J. 1984, Ap. J., 285, L39.

GeRAld EPSTEIN: Office of Technology Assessment, US Congress, Washington, DC 20510

Philip Lubin, George Smoot, and Thyrso Villela: Space Sciences Laboratory and Lawrence Berkeley Laboratory, University of California, Berkeley, CA 94720 